where a human is submerged in an unnatural and constantly changing environment, diving is normally a very safe activity if appropriate training, operating protocols and equipment is used. Serious incidents in diving have been reported as occurring in between $1 / 10000$, and $1 / 2000$ dives, with a fatality seen between $1 / 95000$ and 1/200000. Commercial diving is regulated in the UK by the 'Diving at Work' Regulations (1995) and monitored by the Health and Safety Executive. However, diving has changed dramatically over the years, with new equipment and techniques deployed all the time. Whilst some of this equipment is advantageous (e.g. Wi-Fi enabled dive computers), some has been less so, such as 'build your own Hookah' dive sets. This cheap but bizarre breathing systems, and resulting accidents associated with them in the occupational diving will be explored. Often the Hooka diver does not carry an accessory air source and regulator and the results of an impaired primary air supply precipitate a crisis often in remote and poorly supported working environments. Despite the harvested material from the water being usually sold at high prices to the consumer more and more the diver's remuneration is pitiful and the latter are seeking cheaper ways to work underwater. The Hooka system does not rely on complex equipment but is fraught with danger. Figures from the Royal Hobart Hospital show even excluding cases of carbon monoxide poisoning divers using these systems account for $30 \%$ of the overall hyperbaric treatments for divers. Recent fatal cases along with increasing chamber treatments will be discussed.

\section{C THE OCCUPATIONAL IMPACT OF THE BROAD PRACTICES OF MODERN TUNNELLING}

A Heili. Hyperbaric Tunnelling and Medical Senvices- Romford, UK

\subsection{6/oemed-2018-ICOHabstracts.1066}

The term 'caisson disease' has generally been replaced by dysbaric illness or decompression sickness (DCS) which represent the spectrum of potential problems experienced by those working under unusual pressure. The design and construction of tunnel boring machines (TBMs) are governed not only by soil conditions but now should cater for working at increased pressure and depth. In the right circumstances these machines have reduced not only the intense physical burden on the 'miners' but also limited pressure exposure to interventions into the excavation chamber for inspection and maintenance. Previously to limit ingress of water into the tunnel the whole underground site was kept at pressures above atmospheric. Ground conditions will determine the need for earth pressure balanced or slurry machines. Maintenance on the TBM's cutter head and tools require hyperbaric conditions in the excavation chamber when pressures can exceed 3.5 bar. Deeper tunnelling uses tables and worker surveillance like those used for saturation diving. Breathing mixture is a non-air respirable mixture, such as oxygen and nitrogen (nitrox); oxygen and helium (heliox) or oxygen, nitrogen and helium (trimix) capable of supporting human life under appropriate hyperbaric conditions. Using examples within Egypt, North America and Europe the operational challenges will be expanded with reference to the practical aspects of safe transfer under pressure. Compressed air worker (CAW) is defined as a person certified medically fit for working in compressed air. Aspects of discussions on high pressure compressed air (HPCA) working by
British Tunnelling Society Compressed Air Working Group and the International Tunnelling Association (ITA) will be highlighted. Future developments are likely to attempt to reduce worker exposures to higher pressures by mechanising cutter tool changing.

\section{THE GLOBAL DRIVE TO TACKLE OCCUPATIONAL CANCER}

S Frost. Institution of Occupational Safety and Health (IOSH)

\subsection{6/oemed-2018-ICOHabstracts. 1067}

Introduction This session focuses on the impact of a far-reaching campaign to tackle work-related exposures to carcinogens - and examines what now needs to be done to cut instances of occupational cancer.

Methods Launched by IOSH in 2014, No Time to Lose has raised awareness and understanding of work-related exposure to carcinogens, and helped organisations take action. The campaign has offered free, practical materials for businesses to deliver effective prevention programmes for solar radiation, diesel exhaust emissions and silica dust.

To maximise its impact, IOSH has encouraged organisations to sign the campaign pledge, outlining what they will undertake to manage carcinogenic exposures.

Results Since its launch, the campaign has reached more than 66 million people worldwide through media coverage. More than 200 organisations have formally supported it, with another 100-plus businesses signing up to the pledge. The campaign website has had 207000 visits, with the free resources downloaded 71000 times.

No Time to Lose has been presented at 139 events around the world, in countries including Bulgaria, Cyprus, Egypt and Ireland.

And in November 2016, IOSH, industry leaders, academics and safety and health experts agreed 'Tackling respirable crystalline silica together: a cross-industry commitment', a crosssector plan of action to reduce exposures to this hazardous substance.

Discussion No Time to Lose has raised awareness and understanding of occupational cancer, but carcinogenic exposure remains a widespread threat - an estimated 742000 people die annually from occupational cancer, according to research findings published in 2017.

There are barriers to progress, such as the long latency period between the disease's contraction and symptoms, and attitudinal issues prevalent in male-dominated industries.

This session will be a platform for discussing a global, cross-sector approach to tackling occupational cancer, and explore what delegates can do to promote awareness and help manage its risks among their communities and networks.

\section{MILITARY MEDICINE}

Anthony M Corcoran*. Medical Branch Defence Forces Ireland, Dublin, Ireland

\subsection{6/oemed-2018-ICOHabstracts. 1068}

Aim of special session Outline the development of the Military Medicine specialty, and describe the Irish Naval Service experience of the refugee crisis operations. 